\title{
Do antiviral agents effectively treat Ramsay Hunt syndrome?
}

\author{
Are antiviral agents effective in the treatment of adult patents with \\ Ramsay Hunt syndrome?
}

\section{Uscategui T, Dorée C, Chamberlain IJ, Burton MJ.}

Antiviral therapy for Ramsay Hunt syndrome (herpes zoster oticus with facial palsy) in adults. Cochrane Database Syst Rev 2008;

issue 4

Data sources The Cochrane Ear, Nose and Throat Disorders Group Trials Register, the Cochrane Central Register of Controlled Trials, Medline, PubMed, Embase and other relevant databases (Cinhal. LILACS, KoreaMed, IndMed, PakMediNet, theUK Clinical Research Network Portfolio Database (UKCRN), the World Health Organization International Clinical Trials Registry Platform (ICTRP), Google Scholar, NLH ENT \& Audiology Specialist Library and the metaRegister of Controlled Trials (mRCT).) were utilised to identify possible trials.

Study selection Randomised controlled trials (RCT) were eligible for inclusion if antiviral agents alone or in combination with other therapies (using different routes of administration and dosage schemes) had been taken as treatment for Ramsay Hunt syndrome.

Data extraction and synthesis Two reviewers independently assessed eligibility and trial quality.

Results Only one RCT was identified and included. It was of low quality and included only 15 participants. In this 1992 trial, treatment with intravenous aciclovir and corticosteroids was compared with corticosteroids alone. Analysis found no statistically significant difference between the two groups.

Conclusions The use of antiviral agents against herpes zoster infections in other parts of the body suggests that they could be useful in the case of herpes zoster oticus. We found no evidence that they have a beneficial effect on outcomes in Ramsay Hunt syndrome, despite their widespread use in this condition. As usual, however, the absence of positive evidence of benefit (or, in this case, the 'negative' result of one small, statistically underpowered study) does not necessarily indicate that antivirals are ineffective. The results of the review do suggest that the various adverse effects of antivirals should be taken into consideration in the risk-benefit analysis that foregoes treatment.

\section{Commentary}

Ramsay Hunt syndrome is an uncommon disorder caused by varicella zoster virus (VZV; the cause of chickenpox, also termed herpes zoster). Viral infection of the geniculate ganglion within the temporal bone principally gives rise to vesiculation and ulceration of the external ear and ipselateral anterior two-thirds of the tongue and soft palate, as well as a lower motor neuron palsy of the facial nerve. Because of its rarity, few studies of any design have investigated the most appropriate means of resolving the signs and symptoms of this disorder. Indeed, the present review found only one RCT that evaluated the efficacy of antiviral therapy (with or without corticosteroids) for Ramsay Hunt syndrome.

This report concludes that intravenous aciclovir plus corticosteroids is no more effective than corticosteroids alone in promoting facial nerve recovery at 6 months after onset of disease. As might be expected, the authors conclude that high-quality, appropriately powered RCT are required, and suggest a number of sensible outcomes.

There are increasing numbers of agents available for the treatment of VZV, so any future studies of this rare disorder should take account of current literature detailing their efficacy in the management of VZV. Unless vaccination policies for VZV become widespread there will always be a risk of occasional adults developing Ramsay Hunt syndrome, so there is a clinical need for rigorous studies of therapy. The low prevalence of Ramsay Hunt syndrome means that any future RCT will need to be multicentred in order to obtain the numbers of patients required for a conclusive study. Although these studies will be logistically difficult and expensive, they should provide guidance over the most appropriate means of resolving what is an alarming disorder for affected individuals.

\section{Stephen Porter}

University College London Eastman Dental Institute, London, UK

Evidence-Based Dentistry (2008) 9, 116. doi:10.1038/sj.ebd.6400616 